\title{
The Nonzero External Magnetic Field of Long Solenoids
}

\author{
Shiwei Ye \\ Beijing Computational Science Research Center, Beijing, China \\ Email: leonardo10@163.com
}

How to cite this paper: Ye, S.W. (2022) The Nonzero External Magnetic Field of Long Solenoids. Journal of Electromagnetic Analysis and Applications, 14, 19-30. https://doi.org/10.4236/jemaa.2022.142002

Received: January 15, 2022

Accepted: February 11, 2022

Published: February 14, 2022

Copyright (c) 2022 by author(s) and Scientific Research Publishing Inc. This work is licensed under the Creative Commons Attribution International License (CC BY 4.0).

http://creativecommons.org/licenses/by/4.0/

\begin{abstract}
A widespread assertion has existed for a long time, believing the external field of an infinitely long solenoid should be zero, but it is proofed to be wrong in this work. The components of magnetic flux density of current-carrying, closely wound cylindrical solenoids are calculated. At a distant field point, the external field definitely has a nonzero component, being equal to that of a straight wire of equal length. Since this equivalence is length-independent, it still holds true for ideal solenoids having infinite length. Hence the incorrect and still spreading inference about long solenoids should be rectified. Furthermore, theoretical and experimental discussions involving solenoids should be reviewed again carefully.
\end{abstract}

\section{Keywords}

Solenoid, Magnetic Flux Density, Vector Potential, Aharonov-Bohm Effect

\section{Introduction}

Scientific research related to magnetic field not only has a long history [1] [2], but also covers a wide range of fields [3]. Solenoids and toroids are widely known as basic circuit structures that could generate magnetic field. It is believed that the external magnetic field generated by an infinitely long, closely wound, current-carrying ideal cylindrical solenoid is zero [4]. Moreover, this inference is used as a basic knowledge in further studies, such as the early research works related to the Aharonov-Bohm effect [1] [2]. However, as will be elucidated in this work, the inference about solenoids is wrong. Besides, a similar incorrect inference about the external magnetic field of closely wound toroids also exists [5]. Therefore, the studies involving solenoids and toroids might be affected by these erroneous inferences. 
This paper is devoted to correcting this long-standing and widespread fallacy about long solenoids which is still spreading in textbooks [4] [5], by giving the correct results of the external magnetic field distribution of the ideal solenoids. In this work, the components of the magnetic flux density generated by solenoids are numerically calculated using the Biot-Savart Law. By comparing with the results of numerical calculations, one could notice that there is a universal equivalence between the magnetic field of a solenoid and that of an equally long straight wire located along the solenoid axis, no matter whether the length is infinite. With the length increasing, the calculated field approaches that of an infinitely long solenoid. In this extreme case, the field distribution at distant points turns out to be exactly the same as that of an infinite straight wire.

\section{Calculations and Results}

\subsection{Theoretical Explanations}

A simple transforming process could explain intuitively why the external field of current-carrying solenoids might not be zero. For simplicity, the values are calculated and discussed without any other magnetic media nearby. As shown in Figure 1(a), a highly flexible thin wire is closely wound around a cylinder with cross-section radius $R$, forming a helical line with pitch $H_{0}$. The diameter of the wire, $D_{0}$, is smaller than $H_{0}$. A closely wound solenoid requires $D_{0}<H_{0} \ll R$. When carrying a steady current with intensity $I$, the helical wire forms a solenoid

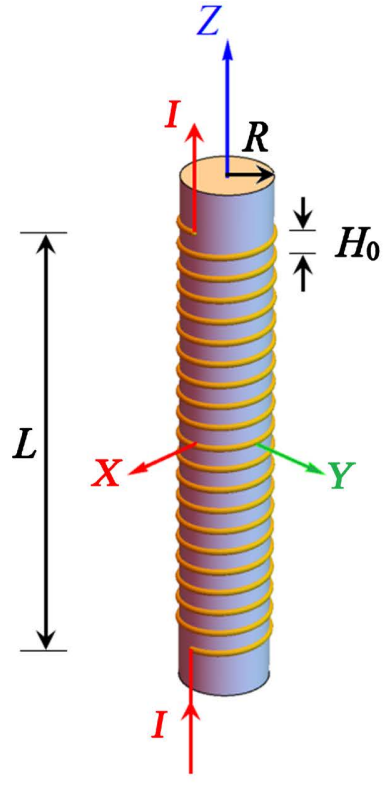

(a)

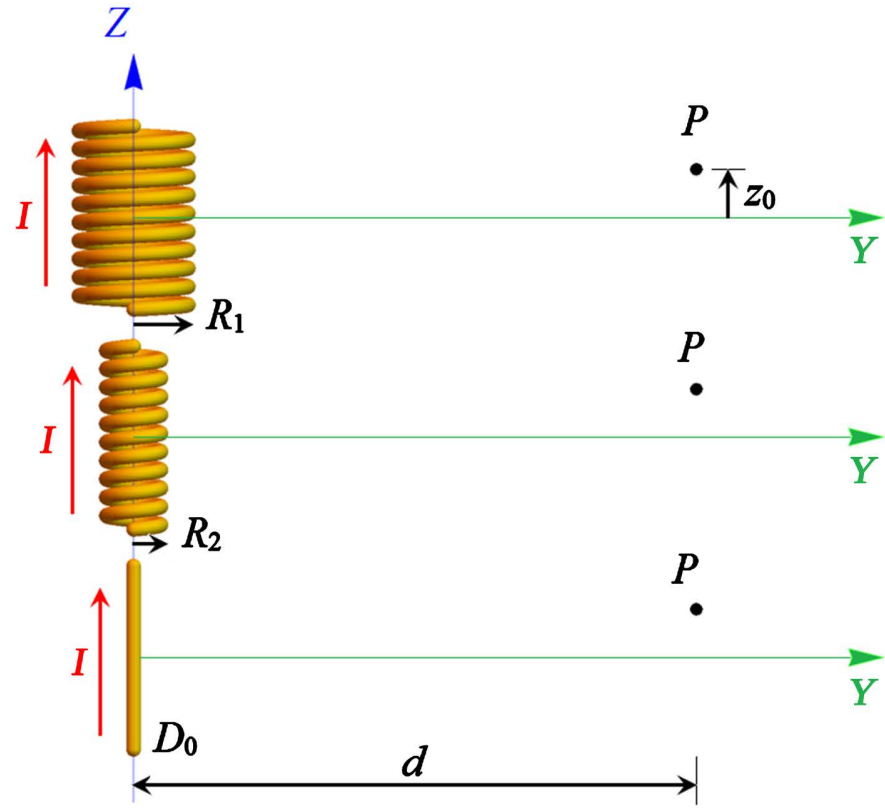

(b)

Figure 1. The structure of a solenoid and the transforming process. (a) The parameters of the solenoid. A flexible thin wire is wound around a cylinder with radius $R$, forming a solenoid with total length $L$ and pitch $H_{0}$. (b) The transforming process from a solenoid to a straight wire. The diameter of the wire, $D_{0}$, is smaller than $H_{0}$. The steady current intensity in each wire is $I . P$ is a distant field point at $\left(0, d, z_{0}\right)$. 
with total length $L$. Then the winding turns per unit length of this solenoid is, $n$ $=1 / H_{0}$. The axis of the cylinder is set as the $Z$ axis. The distance from a field point, $P$, to $Z$-axis is $d$. The perpendicular line from $P$ to $Z$-axis is parallel to the $Y$ axis.

As shown in Figure 1(b), for an infinitely long solenoid, let the radius $R$ decrease gradually and slowly. The solenoid keeps shrinking and getting thinner and thinner. Finally, when its radius $R$ becomes less than the radius of the wire $\left(R<D_{0} / 2\right)$, the solenoid can be recognized as a straight wire. During this transforming process, the external magnetic field of the solenoid at $P$ changes continuously and finally becomes the field of an infinite straight wire, which is nonzero apparently. This indicates that the characteristic of the external field of a solenoid is closely related to that of a straight wire. Especially, it inspires us to speculate that the external field of an infinite solenoid might also be nonzero.

In general, the detailed characteristics of the wire-shape localized within the scale of $R$, should not play an important role in determining the magnetic field at a far away point $P(d \gg R)$. The overall magnetic field distribution of a very thin solenoid should be very close to that of a simple straight wire. On the other hand, when a straight wire changes its structure (such as bending or twisting) around a certain spot localized within the scale of $R$, the corresponding change of its field could not be reflected significantly at those distant points.

Theoretically, each elementary current $I \mathrm{~d} l$ of the wire could be decomposed into three components, $\mathrm{d} \boldsymbol{l}_{z}$ (paralleling to $Z$-axis), $\mathrm{d} \boldsymbol{l}_{T}$ (along the tangential direction of the cross-section circumference), and $\mathrm{d} I_{R}$ (along the radial direction of the circle). $\quad \mathrm{d} \boldsymbol{l}=\mathrm{d} \boldsymbol{I}_{z}+\mathrm{d} \boldsymbol{I}_{\tau}+\mathrm{d} \boldsymbol{I}_{R}$. Since the radius $R$ is fixed here for solenoids, and the diameter of the wire $D_{0}$ is negligible compared to $R\left(D_{0}<H_{0} \ll R\right)$, $\mathrm{d} \boldsymbol{I}_{R}$ equals zero. Then we have

$$
\mathrm{d} \boldsymbol{l}=\mathrm{d} \boldsymbol{I}_{z}+\mathrm{d} \boldsymbol{l}_{\tau}
$$

The infinitesimal, $\mathrm{d} \boldsymbol{B}$, of the field generated by $I \mathrm{~d} \boldsymbol{I}$ at a point $P$ is given by the Biot-Savart Law,

$$
\mathrm{d} \boldsymbol{B}=\left(\frac{\mu_{0}}{4 \pi}\right) I \frac{\mathrm{d} \boldsymbol{l} \times \boldsymbol{r}}{r^{3}}
$$

Then the magnetic flux density $\boldsymbol{B}$ generated by the current-carrying wire, would be the integration of $\mathrm{d} \boldsymbol{B}$ along the wire,

$$
\boldsymbol{B}=\int \mathrm{d} \boldsymbol{B}=\left(\frac{\mu_{0}}{4 \pi}\right) I \int \frac{\mathrm{d} \boldsymbol{l}_{z} \times \boldsymbol{r}}{r^{3}}+\left(\frac{\mu_{0}}{4 \pi}\right) I \int \frac{\mathrm{d} \boldsymbol{l}_{\tau} \times \boldsymbol{r}}{r^{3}}
$$

At a distant point $P$, the first term is approximately equal to the field of a straight wire with the same length of the solenoid, while the second term is approximately equal to that of a series of circular currents with radius $R$, arranged at equal intervals along the $Z$ axis. If the total length $L$ is infinite, then the second term is zero, while the first term is not. Therefore, the result of $B$ at $P$ is approximately equal to that generated by an infinitely long straight wire.

As for toroids, a similar assertion exists, believing that their external magnetic 
field is zero [5]. Based on a similar analysis as the transforming process, it is easy to know the truth that at distant points, the field is similar to that of a magnetic dipole. At nearer points, the field is close to that of a circular current or a donut-shaped current distribution. In this work, we focus on solenoids mainly, while toroids will be discussed elsewhere.

In the following section, the magnetic field distribution of cylindrical solenoids will be discussed with detailed calculations and analyses.

\subsection{Parameters and Descriptions of the Calculations}

Let the expression of the helical wire be

$$
\begin{aligned}
& x=R \cos (\alpha) \\
& y=R \sin (\alpha) \\
& z=\left(\frac{H_{0}}{2 \pi}\right) \alpha
\end{aligned}
$$

where $\alpha$ is an independent variable for determining the position of each elementary current, Id $l$. An $I \mathrm{~d} l$ could be expressed by the differentials of the coordinates,

$$
I \mathrm{~d} \boldsymbol{I}=I(\mathrm{~d} x, \mathrm{~d} y, \mathrm{~d} z)=\left(-R \sin (\alpha), R \cos (\alpha), \frac{H_{0}}{2 \pi}\right) I \mathrm{~d} \alpha
$$

The position of the field point $P$ is at $\left(x_{P}, y_{P}, Z_{P}\right)$. Set $x_{P}=0$ for simplicity. As mentioned earlier, the distance from $P$ to $Z$-axis, $d$, equals $y_{P}$. Then we have

$$
\begin{aligned}
& x_{P}=0 \\
& y_{P}=d \\
& z_{P}=z_{0}
\end{aligned}
$$

where $Z_{0}$ is another variable for determining the position of $P$.

The following calculations are based on the Biot-Savart Law. In Equation (2a), $I$ is the current intensity in the wire, $\boldsymbol{r}$ is the position vector pointing from elementary current $I \mathrm{~d} I$ to $P$, and $r$ is the module of $r$.

$$
\begin{gathered}
\boldsymbol{r}=\boldsymbol{r}_{P}-\boldsymbol{r}_{\mathrm{Id} l}=\left(-R \cos (\alpha), d-R \sin (\alpha), z_{0}-\frac{H_{0}}{2 \pi} \alpha\right) \\
r=|\boldsymbol{r}|=\sqrt{[-R \cos (\alpha)]^{2}+[d-R \sin (\alpha)]^{2}+\left(z_{0}-\frac{H_{0}}{2 \pi} \alpha\right)^{2}}
\end{gathered}
$$

The result of the outer product could be expanded as,

$$
\begin{aligned}
\mathrm{d} \boldsymbol{l} \times \boldsymbol{r} & =\boldsymbol{u}(\alpha) \mathrm{d} \alpha \\
& =\left[\begin{array}{c}
\boldsymbol{i} \\
\boldsymbol{j} \\
\boldsymbol{k}
\end{array}\right]^{\mathrm{T}}\left[\begin{array}{c}
-\frac{H_{0} d}{2 \pi}-\frac{\alpha H_{0} R \cos (\alpha)}{2 \pi}+R z_{0} \cos (\alpha)+\frac{H_{0} R \sin (\alpha)}{2 \pi} \\
-\frac{H_{0} R \cos (\alpha)}{2 \pi}-\frac{\alpha H_{0} R \sin (\alpha)}{2 \pi}+R z_{0} \sin (\alpha) \\
R^{2}-R d \sin (\alpha)
\end{array}\right] \mathrm{d} \alpha
\end{aligned}
$$


where $\boldsymbol{u}(\alpha)$ is a vector function of variable $\alpha$. The magnetic flux density at $P$ is given by the integration of $\mathrm{d} \boldsymbol{B}$,

$$
\boldsymbol{B}=\int \mathrm{d} \boldsymbol{B}=\left(\frac{\mu_{0}}{4 \pi}\right) I \int \frac{\mathrm{d} \boldsymbol{l} \times \boldsymbol{r}}{r^{3}}=\int_{\alpha_{1}}^{\alpha_{2}} \boldsymbol{f}(\alpha) \mathrm{d} \alpha,
$$

where the vector function $f(\alpha)$ could be expressed as,

$$
\boldsymbol{f}(\alpha)=\left(\frac{\mu_{0}}{4 \pi}\right) I \frac{\boldsymbol{u}(\alpha)}{r(\alpha)^{3}} .
$$

In a practical calculation, the integration could be replaced by the following summation with a properly selected $\Delta \alpha$,

$$
\boldsymbol{B}=\sum_{\alpha=\alpha_{1}}^{\alpha_{2}} \boldsymbol{f}(\alpha) \Delta \alpha
$$

\subsection{Results and Comparisons}

In the following calculations, the steady current in the wire is known as $I$. The unit of length is selected as the pitch of the helical wire $H_{0}$, while the unit of $\boldsymbol{B}$ is $\frac{\mu_{0} I}{4 \pi H_{0}}$. Then $B$ is related to several variables.

$$
\boldsymbol{B}=\boldsymbol{B}\left(L, d, R, z_{0}\right) .
$$

The meanings of the variables are listed earlier in Section 2.1. In this section, the relation between $\boldsymbol{B}$ and each variable is discussed respectively.

The $B_{X}$ component of the external field of a solenoid is always compared to that of a straight wire with the same length and current intensity. Since $z_{0}$ is a variable, it is convenient to set the origin at the middle of the solenoids or the straight wires, without losing generality. The field of a straight wire, $\boldsymbol{B}_{L}$, only has one non-zero component, $B_{L x}$ the magnitude of which is

$$
B_{L}=\left|\boldsymbol{B}_{L}\right|=\left|B_{L x}\right|=\left(\frac{\mu_{0}}{4 \pi}\right) \frac{I}{d}\left[\frac{L / 2+z_{0}}{\sqrt{\left(L / 2+z_{0}\right)^{2}+d^{2}}}+\frac{L / 2-z_{0}}{\sqrt{\left(L / 2-z_{0}\right)^{2}+d^{2}}}\right],
$$

where $L$ is also the length of the wire, while other variables are the same as those mentioned earlier. The theoretical values of $B_{L x}$ are used as the standards to compare with the calculated values of $B_{X}$ of the solenoids. An extremely small relative difference between $B_{X}$ and corresponding $B_{L X}$ would show their close relevance.

\subsubsection{The Relationship between $B$ and $L$, the Total Length of the Solenoid}

Figure 2 shows the calculated $B_{x}$ of a solenoid and the $B_{L x}$ of a corresponding straight wire, while the fixed parameters are as follows, $R=100 H_{0}, d=10,000 H_{0}$ $=100 R$, and $z_{0}=0$. The selection of parameters here ensures that $H_{0} \ll R \ll d$. A zero $z_{0}$ makes the results compatible to the symmetry of the infinite solenoid case, as $L \rightarrow \infty$.

It is verified by the calculation that, at a fixed distant field point $P$ $\left(d=100 R \gg R\right.$ ), the $B_{X}$ of the solenoid is always equal to the $B_{L x}$ of the corresponding straight wire placed along $Z$-axis with an equal length, $L$, no matter 


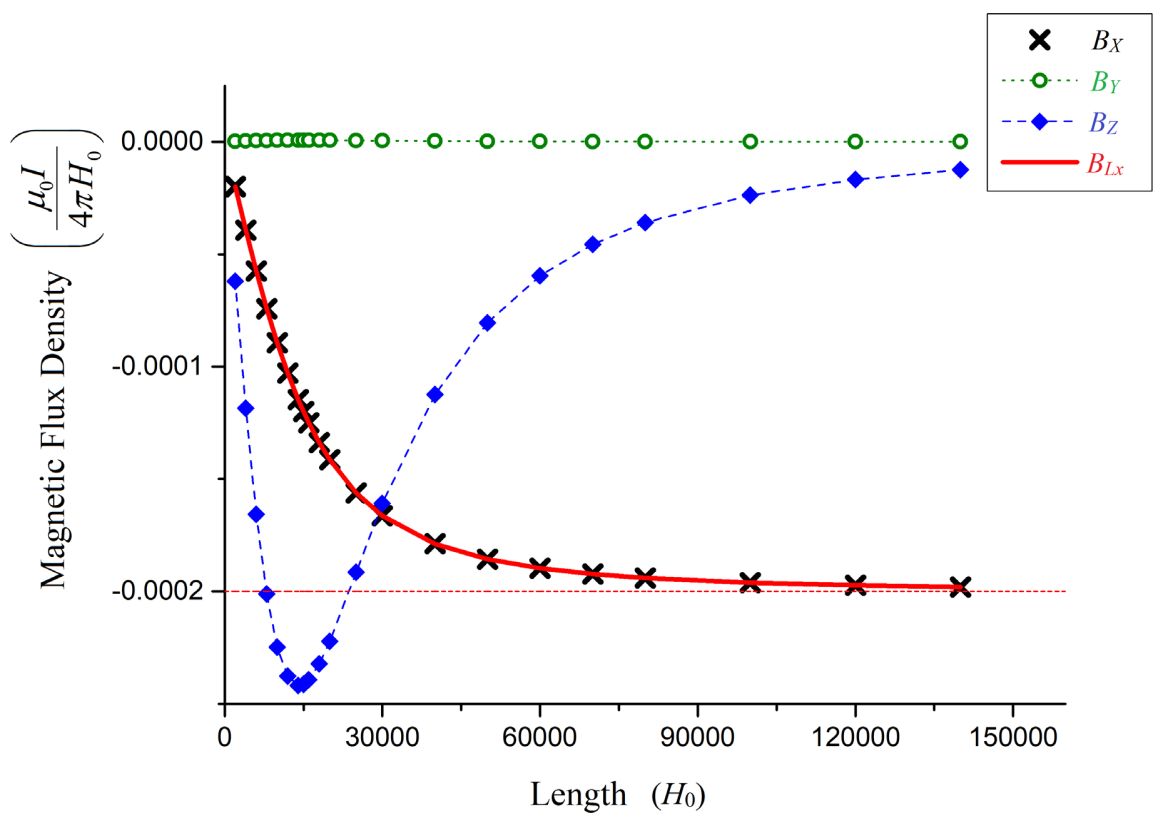

Figure 2. The components of the external magnetic flux density of a solenoid at a distant point, as functions of the solenoid's length $L$. (The parameters are as follows, $R=100 H_{0}$, $d=10,000 H_{0}=100 R$, and $z_{0}=0$. The red line represents the theoretical values of the corresponding straight wire, $B_{L x}$, while the black marks show the calculated values of $B_{X}$ for the solenoid with Equation (7). The relative difference between each $B_{X}$ and the corresponding $B_{L X}$ is less than $1 \times 10^{-3}$. The blue line represents the calculated values of $B_{Z}$, while the green line represents those of $B_{Y}$. For $L>15,000 H_{0}, B_{Z}$ decreases gradually to zero when $L$ increases to infinity.)

what the value of $L$ is. The relative difference between them is less than $1 \times 10^{-3}$. Moreover, when $L$ increases, the absolute value of $B_{X}$ keeps increasing. As $L$ approaches infinity, $\left|B_{X}\right|$ approaches its maximum limit, being exactly the value of the magnetic field of an infinite straight wire,

$$
B_{L \rightarrow \infty}=\frac{\mu_{0} I}{2 \pi d}=\left(\frac{\mu_{0} I}{4 \pi H_{0}}\right) \frac{2 H_{0}}{d}=\left(\frac{\mu_{0} I}{4 \pi H_{0}}\right) \times 0.0002 .
$$

For those long enough solenoids (with $L>150 R=15,000 H_{0}$ ), the absolute value of $B_{z}$ keeps decreasing when $L$ increases. As $L$ approaches infinity, $\left|B_{z}\right|$ approaches zero. This could be expected by the picture of the projected magnetic induction lines on the $Y O Z$-plane of solenoids with finite length.

$B_{y}$ approximately remains zero at a point with $Z_{0}=0$, when $L$ changes. It would be shown in the following section 2.3.3 that this result is in accord with the symmetric characteristic of the magnetic field.

\subsubsection{The Relationship between $B$ and $d$, the Distance from Point $P$ to the Solenoid Axis}

Figure 3 shows the calculated components of $\boldsymbol{B}$, as functions of distance $d$. The fixed parameters are as follows, $R=100 H_{0}, L=20,000 H_{0}=200 R$, and $z_{0}=0$.

The value of $B_{X}$ of the solenoid still fits very well to the corresponding $B_{L x}$. Their relative difference is less than $1 \times 10^{-3}$. Actually, the value of $B_{X}$ matches 


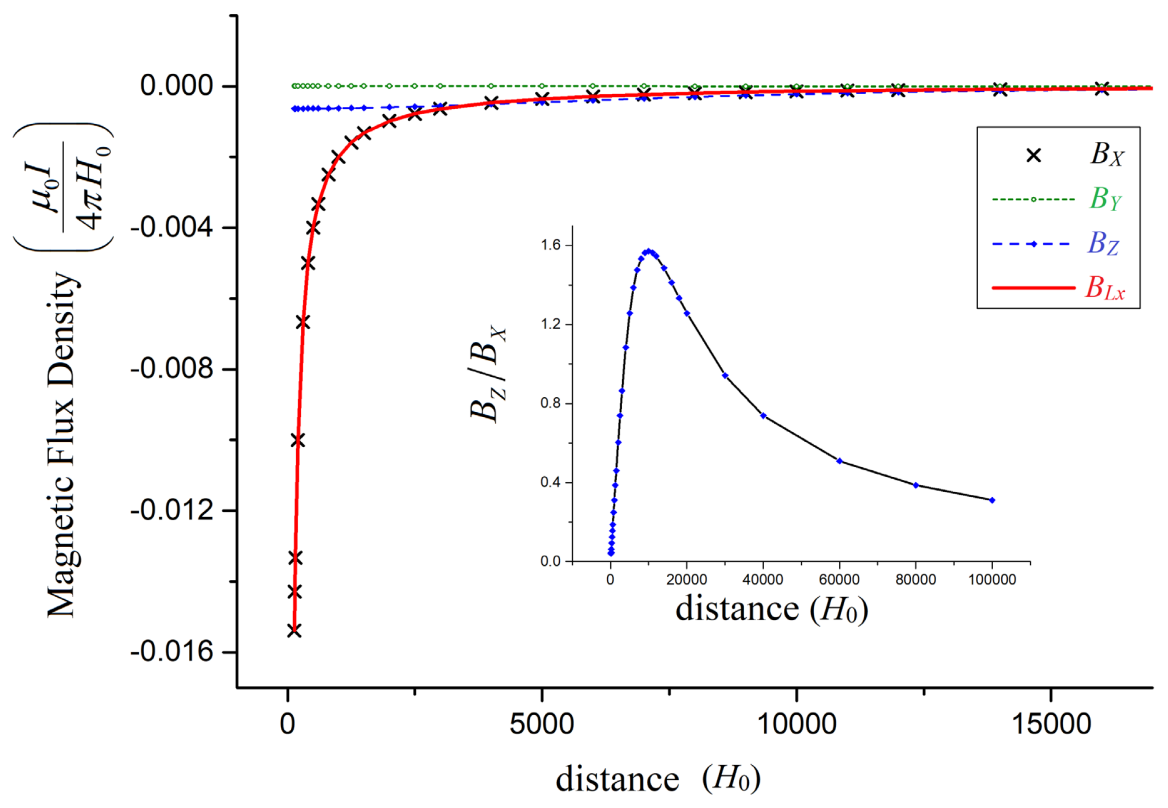

Figure 3. The relation between the external $B$ of a solenoid and the distance $d$. (The inset of Figure 3 shows the comparison of $B_{Z}$ with corresponding $B_{X}$. The parameters are as follows, $R=100 H_{0}, L=20,000 H_{0}=200 R$, and $\left.z_{0}=0\right)$.

the corresponding $B_{L x}$ quite well with any $d>1.3 R$, although the general requirement is $d \gg R$. This is because that at a field point not so far away, the first term of Equation (2b) is approximately equal to the field of a cylindrical current distribution with radius $R$, whose external field is exactly the same as a straight wire.

The magnitudes of $B_{X}$ and $B_{Z}$ are both decreasing as the distance $d$ increasing. This could be expected, since the field becomes weaker at a further point, in general. As shown in the inset of Figure 3, when $d>100 R=10,000 H_{0}$, with the increase of $d$, the ratio $\left|B_{Z}\right| /\left|B_{X}\right|$ gradually decreases and approaches zero.

Again, the magnetic induction lines projected on the YOZ-plane of a solenoid indicates that $B_{Y}=0$ at a point with $z_{0}=0$, since the solenoid is approximately symmetric about the origin.

\subsubsection{The Components of $B$ as Functions of $z_{0}$}

Figure 4 shows the relation between $\boldsymbol{B}$ and $z_{0}$, the $z$ coordinate of the field point. The fixed parameters are as follows, $R=100 H_{0}, L=20,000 H_{0}=200 R$, and $d=$ $10,000 H_{0}=100 R$.

It is clear that $z_{0}$ can affect all three components of $\boldsymbol{B}$ significantly. $B_{X}$ and $B_{Z}$ are even functions of $z_{0}$, while $B_{Y}$ is an odd function of $z_{0}$. Here we can see clearly that $B_{Y}$ always equals zero at those points with $z_{0}=0$, because of the symmetry of the field distribution, as mentioned earlier. When $z_{0}=0$, both $B_{X}$ and $B_{Z}$ reach their respective minima, while their magnitudes reach the respective maxima.

Again, at all the distant field points, the values of $B_{X}$ of the solenoid consistently match those of $B_{L x}$ of the equally long straight wire. The relative difference between them is less than $1 \times 10^{-3}$. The values of $B_{Y}$ and $B_{Z}$, are in conformity 
with the field distribution on the YOZ-plane illustrated by the projected magnetic induction lines of a finite solenoid.

\subsubsection{The Components of $B$ with Different Radius $R$}

The fixed parameters are as follows, $z_{0}=0, L=20,000 H_{0}$, and $d=10,000 H_{0}$. As shown in Figure 5 , the tightness of winding, represented by $\left(R / H_{0}\right)$, hardly affects

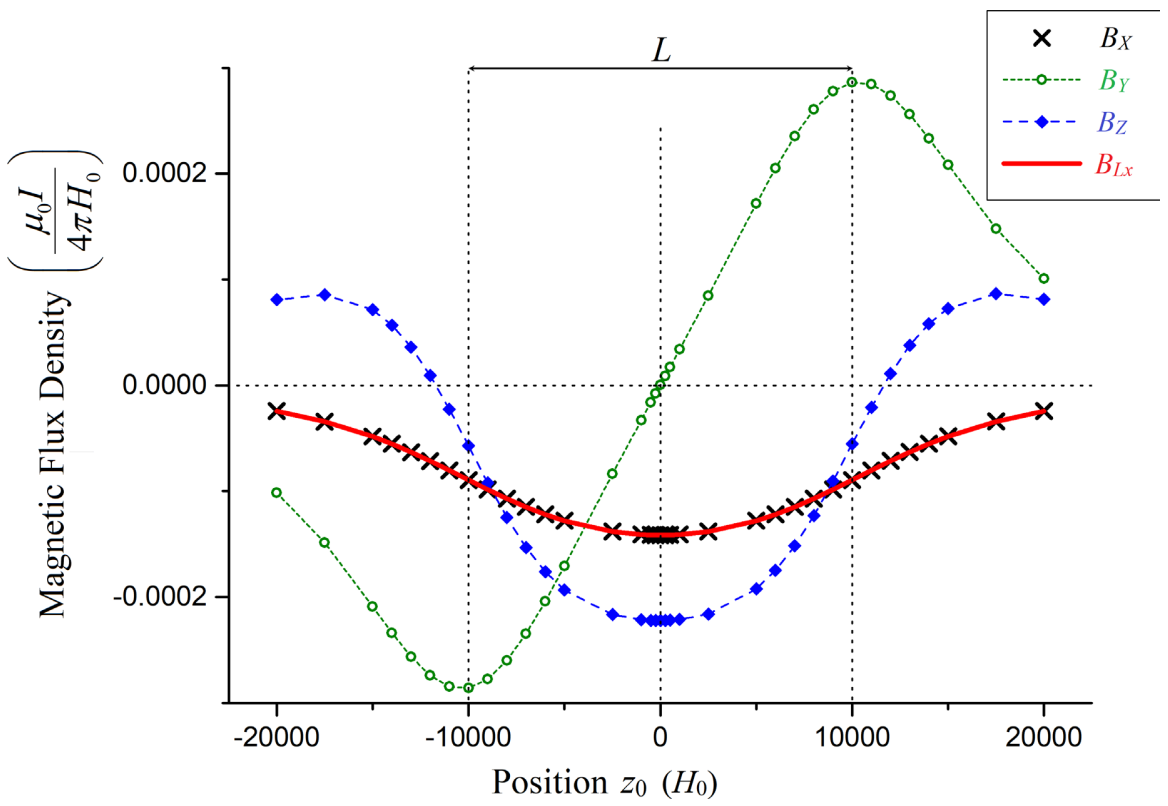

Figure 4. The components of $B$ of a solenoid, as functions of $Z_{0}$. (The parameters are as follows, $R=100 H_{0}, L=20,000 H_{0}=200 R$, and $d=10,000 H_{0}=100 R$. The red line represents the theoretical values of the corresponding straight wire, $B_{L x}$. The relative difference between $B_{X}$ and $B_{L X}$ is less than $1 \times 10^{-3}$.)

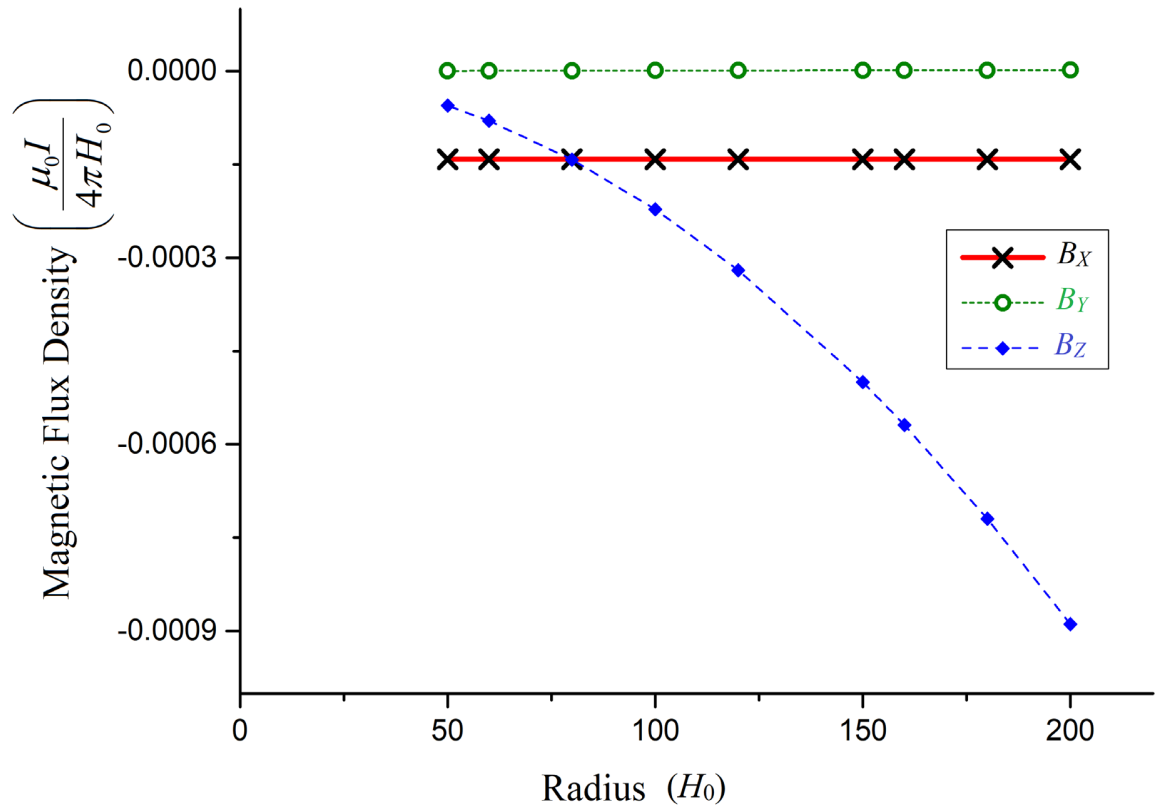

Figure 5. The relation between $B$ and the solenoid's radius $R$. (The parameters are as follows, $Z_{0}=0, L=20,000 H_{0}$, and $d=10,000 H_{0}$.) 
the value of $B_{X}$ at a distant point, though $B_{X}$ is closer to the value of a straight wire with a lesser $R$, obviously. The relative difference between the maximum and the minimum of $B_{X}$ is less than $1 \times 10^{-4}$.

On the contrary, the radius $R$ of the solenoid affects $B_{Z}$ more significantly, because the field distribution on the $Y O Z$-plane is definitely related to the radius of the solenoid with finite length. In fact, at those points where $Z_{0}$ is not zero, both $B_{Y}$ and $B_{Z}$ will be influenced by the value of $R$, while all the values of $B_{Y}$ here approximately equal zero because a symmetric field point with $z_{0}=0$ is chosen.

\subsubsection{Summary of the Results and Comparisons}

Since adequate discussions have been made for solenoids with finite length, it is possible to infer the results for a very long solenoid.

It has been verified that, no matter how long the solenoid is, the $B_{X}$ of its external field always equals that of a straight wire having an equal length and carrying an equal current. This equivalence itself is independent of the value of length $L$. Therefore, at a distant point, an infinitely long cylindrical solenoid would generate a nonzero external magnetic field, with its $B_{X}$ component being equal to that of an infinite straight wire, i.e.

$$
\left|B_{x}\right|=\frac{\mu_{0} I}{2 \pi d}
$$

On the other hand, according to the symmetry of the infinite solenoid, $B_{Y}$ equals zero. Since the length $L$ is infinity, $B_{Z}$ also equals zero. Therefore, both $B_{Y}$ and $B_{Z}$ of the external field are zero. Then the magnitude of the external magnetic flux density of an infinitely long solenoid at a distant field point is $B=\left|\boldsymbol{B}_{\text {ext }}\right|=\left|B_{x}\right|=\frac{\mu_{0} I}{2 \pi d}$. Naturally, the external $\boldsymbol{B}_{\text {ext }}$ satisfies the Ampere's law,

$$
\oint \boldsymbol{B}_{\mathrm{ext}} \cdot \mathrm{d} \boldsymbol{I}=\mu_{0} I,
$$

where $I$ is exactly the flux of the current density through an arbitrary cross-section of the solenoid, i.e., the current enclosed by the Amperian loop parallel to the $X O Y$-plane.

\section{The Substitution of Solenoids}

We have proofed that, no matter how long it is, a solenoid always has an external nonzero $B_{X}$ component. So the problem is finding a substitution of solenoid. The answer is simple. A pair of coaxial but chiral-opposite solenoids can build a field-free area enclosing a nonzero amount of magnetic flux.

As for the Aharonov-Bohm effect [1], Figure 6(a) shows the schematic experiment observing the inference of two beams of electron passing through a field-free area (with $\boldsymbol{B}=\mathbf{0}$ ) enclosing a nonzero flux amount. Many experiments have been established to measure the results of the Aharonov-Bohm effect, i.e., the change of inference fringes [2] [6]. In fact, to distinguish the effects of $\boldsymbol{A}$ and $B$, one can just make these two variables change conversely, in the area where the beams pass through. 


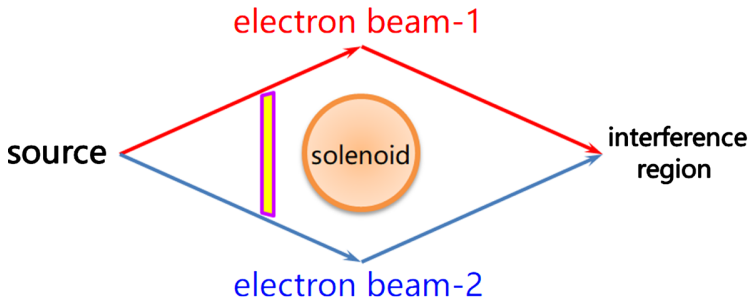

(a)

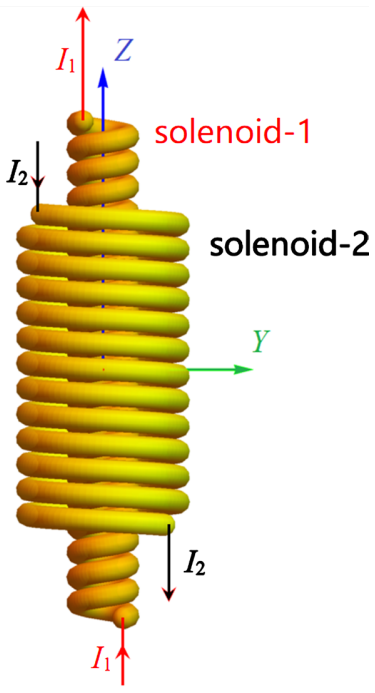

(b)

Figure 6. A substitution of a solenoid. (a) Schematic experiment to demonstrate the Aharonov-Bohm effect. (b) A pair of two coaxial solenoids with opposite chirality.

\subsection{Coaxial, Chiral-0pposite Solenoids Carrying Equal Steady Currents}

As shown in Figure 6(b), suppose there are two coaxial solenoids, with opposite chirality and different cross-section radius, carrying steady current with intensity,

$$
I_{1}=I_{2}=I .
$$

If the $Z$-components of their current density are in opposite directions, then their internal fields are mutually reinforcing, while their external fields are canceling each other out. Thus, the structure of an external field-free area where the beams passing through with nonzero magnetic flux enclosed between the two beams is ideally realized.

\subsection{Coaxial, Chiral-0pposite Solenoids Carrying Slowly Varying Currents}

The device mentioned in Section 3.1 can be used as a substitution of a solenoid in certain cases, such as the experiments related to the Aharonov-Bohm effect. Though the leakage of field of the solenoid has been discussed in other works [2] [7], only the $B_{Z}$ component is considered, while the $B_{X}$ component is ignored, even the external $\boldsymbol{B}_{X}$ is parallel to the vector potential $\boldsymbol{A}_{\text {int }}$ of the internal $\boldsymbol{B}$ of the solenoid.

In this section, we don't need $B$ to be zero on the routes of the beams. On the contrary, we let both $\boldsymbol{B}$ and $\boldsymbol{A}$ exist together in the same area, changing conversely. It is only necessary to distinguish which variable can affect the phase difference, the magnetic flux in the area surrounded by the routes related to vector $\boldsymbol{A}$, or the fields along the routes related to vector $\boldsymbol{B}$. Therefore, the existence of the field-free areas is not a necessity, if the changing trend of $B$ is opposite to that of $A$. 
In the first step, let the current intensity in solenoid- 1 be zero, $I_{1}=0$, and that in solenoid-2, $I_{2}$, increase very slowly. Then the magnetic flux increase gradually and may cause a changing trend $T_{1}$ of the interference fringes.

In the second step, fix $I_{2}$ and let $I_{1}$ increase very slowly. The magnetic flux generated by both currents increases gradually, while the field outside solenoid-2 decreases gradually. The changing trend of the interference fringes in this step is noted as $T_{2}$.

By comparing $T_{1}$ and $T_{2}$, it could be distinguished whether the change of interference fringes is caused by the magnetic flux or by the magnetic field itself. In these steps, the changing trends of the flux are the same, while those of the field intensity are opposite. If $T_{1}$ and $T_{2}$ always show the same tendency, then the change of flux dominates this process. Otherwise, the change of field intensity may have played an important role in this process.

\section{Summary}

The assertion that the external field of a very long solenoid is zero has been proved to be wrong. Although it directly violates the Ampere's law and the Biot-Savart Law, this fallacy is still spreading widely.

The occurrence and spread of this fallacy might be due to the following reasons. A possible theoretical reason might be the improper substitution of a series of coaxial circular currents for the original solenoid, which changes the symmetric properties of the system and totally ignores the contribution of the $\mathrm{d}_{z}$-term to $B$ in Equation (2b). A possible experimental reason might be the truth that the external field is much weaker than the internal field of a solenoid.

$$
B_{\text {ext }}=\frac{\mu_{0} I}{2 \pi d}<\frac{\mu_{0} I}{2 \pi R} \ll \frac{\mu_{0} I}{2 \pi H_{0}}<\frac{\mu_{0} I}{H_{0}}=B_{\mathrm{int}}
$$

The difference of intensity would become more significant when ferromagnetic medium is inserted inside the solenoid. Besides, only the magnetic induction lines projected on the YOZ-plane of solenoids are concerned and shown in experiments, while those projected on the $X O Y$-plane are ignored.

In this work, the external magnetic field flux density of solenoids is calculated numerically. The results of $B_{X}$ are compared with the $B_{L X}$ of the corresponding straight wires. The universal equivalence between $B_{X}$ and $B_{L x}$ is shown. Therefore, the assertion about long solenoids is rectified. A structure of coaxial pair of solenoids is proposed as a substitution of solenoid to create an external field-free area. Theoretical and experimental discussion related to solenoids and toroids might be revised.

\section{Acknowledgements}

The author would like to thank Professor Bang-jiao Ye for the valuable guidance and insightful comments, and Professor Si-zhao Jin for the long-term, tireless help and support. 


\section{Conflicts of Interest}

The author declares no conflicts of interest regarding the publication of this paper.

\section{References}

[1] Aharonov, Y. and Bohm, D. (1959) Significance of Electromagnetic Potentials in the Quantum Theory, Physical Review, 115, 485-491. https://doi.org/10.1103/PhysRev.115.485

[2] Chambers, R.G. (1960) Shift of an Electron Interference Pattern by Enclosed Magnetic Flux. Physical Review Letters, 5, 3-5. https://doi.org/10.1103/PhysRevLett.5.3

[3] Drochon, A., Robin, V., Fokapu, O. and Rodriguez, D. (2016) Stationary Flow of Blood in a Rigid Vessel in the Presence of an External Magnetic Field: Considerations about the Forces and Wall Shear Stresses. Applied Mathematics, 7, 130-136. https://doi.org/10.4236/am.2016.72012

[4] Feynman, R.P., Leighton, R.B. and Sands, M.L. (2010) The Feynman Lectures on Physics, Vol. 2. Chapter 14-4. Addison-Wesley, Reading.

[5] Griffiths, D.J. (2012) Introduction to Electrodynamics. Addison-Wesley, New York, Chapter 5 .

[6] Tonomura, A., et al. (1986) Evidence for Aharonov-Bohm Effect with Magnetic Field Completely Shielded from Electron Wave. Physical Review Letters, 56, 792-795. https://doi.org/10.1103/PhysRevLett.56.792

[7] Roy, S.M. (1980) Condition for Nonexistence of Aharonov-Bohm Effect. Physical Review Letters, 44, 111-114. https://doi.org/10.1103/PhysRevLett.44.111 